\title{
Autologous Anti-CD19 T-cell Receptor T cells ET190L1
}

National Cancer Institute

\section{Source}

National Cancer Institute. Autologous Anti-CD19 T-cell Receptor T cells ET 190L1. NCI

Thesaurus. Code C148030.

Autologous human peripheral blood T-lymphocytes transduced with a lentivirus encoding a proprietary expression construct composed of a T-cell receptor (TCR)-like human antibody, which is synthesized by a proprietary phage display platform, targeting peptides derived from the tumor-associated antigen (TAA) CD19 that are presented in the context of major histocompatibility complex (MHC) molecules, with potential antineoplastic activity. Following leukapheresis, isolation of lymphocytes, expansion ex vivo, transduction, and re-introduction into the patient, the autologous anti-CD19 TCR T-cells ET190L1 target and bind to tumor cells expressing CD19 peptide/MHC complexes. This results in cytotoxic T-lymphocyte (CTL)-mediated elimination of CD19-positive tumor cells. CD19, cluster of differentiation antigen 19, is a B-cell-specific cell surface antigen overexpressed in B-cell lineage malignancies. ET 190L1 is able to match the anticancer activity of chimeric antigen receptor (CAR) T-cells; however, ET 190L1 is less likely to stimulate cytokine release syndrome (CRS) and does not cause CAR T-cell-triggered neurotoxicity. 